\title{
CHRONOLOGICAL LIST OF POPES \\ - TENTH AND ELEVENTH CENTURIES
}

John X

Leo VI

Stephen VII

John XI

Leo VII

Stephen VIII

Marinus II

Agapitus II

John XII

Leo VIII

Benedict $\mathrm{V}$

John XIII

Benedict VI

Boniface VII (anti-pope)

Benedict VII

John XIV

John XV

Gregory V

John XVI (anti-pope)

Silvester II

John XVII

John XVIII

Sergius IV

Benedict VIII

Gregory VI (anti-pope)

John XIX

Benedict IX

Silvester III (anti-pope)

Gregory VI

Clement II

Damasus II

Leo IX

Victor II

Stephen IX
Mar./Apr. 914-deposed May 928; d. 929 May-Dec. 928

Dec. $928-F e b .931$ Feb./Mar. 931-Dec. 935/Jan. 936 Jan. 936-July 939 July 939-Oct. 942 Oct. $942-$ May 946 May 946-Dec. 955

Dec. $955^{-M a y} 964$

Dec. 963 -Mar. 965 May 964-deposed June 964; d. 966

Oct. 965-Sept. 972 Jan. 973-July 974 June-July 974 Oct. 974-July 983 Dec. 983-Aug. 984 Aug. 985-Mar. 996 May 996-Feb. 999 Feb. 997-May 998 Apr. 999-May 1003 May-Nov. 1003 Dec. 1003-June/July 1009 July 1009-May 1012 May 1012-Apr. 1024 May-Dec. 1012 Apr. 1024-Oct. 1032 Oct. 1032-May 1045; Nov. 1047-July 1048; d. 1055/56 Jan.-Mar. 1045; d. 1063 May 1045-deposed Dec. 1046; d. 1047

Dec. 1046-Oct. 1047 July-Aug. 1048 Feb. 1049-Apr. 1054 Apr. 1055-July 1057 Aug. 1057-Mar. 1058

xii 
Benedict X (anti-pope)

Nicholas II

Alexander II

Honorius II (anti-pope)

Gregory VII

Clement III (anti-pope)

Victor III

Urban II

Paschal II
Apr. 1058-Jan. 1059; d. after 1073 Dec. 1058-July 1061 Sept. 1061-Apr. 1073 Oct. 1061-May 1064; d. 1071/72 Apr. 1073-May 1085 June 1080; Mar. 1084-Sept. 1100 May 1086; May-Sept. 1087 Mar. 1088-July 1099 Aug. 1099-Jan. 1118 